PROCEEDINGS OF THE

AMERICAN MATHEMATICAL SOCIETY

Volume 139, Number 8, August 2011, Pages 2663-2669

S 0002-9939(2010)10702-5

Article electronically published on December 22, 2010

\title{
NILPOTENCY OF NORMAL SUBGROUPS HAVING TWO G-CLASS SIZES
}

\author{
ELENA ALEMANY, ANTONIO BELTRÁN, AND MARÍA JOSÉ FELIPE
}

(Communicated by Jonathan I. Hall)

\begin{abstract}
Let $G$ be a finite group. If $N$ is a normal subgroup which has exactly two $G$-conjugacy class sizes, then $N$ is nilpotent. In particular, we show that $N$ is abelian or is the product of a $p$-group $P$ by a central subgroup of $G$. Furthermore, when $P$ is not abelian, $P /(\mathbf{Z}(G) \cap P)$ has exponent $p$.
\end{abstract}

\section{INTRODUCTION}

Let $G$ be a finite group and $N$ a normal subgroup of $G$. Since $N$ is a union of $G$-conjugacy classes, it is natural to wonder what information on the structure of $N$ can be obtained from its $G$-class sizes. One result of N. Itô claims that any finite group having exactly two conjugacy class sizes is nilpotent (8]). If every $G$-conjugacy class contained in $N$ has only two possible sizes, 1 or $m$, then is $N$ contained in $\mathbf{F}(G)$, the Fitting subgroup of $G$ ? We remark that the fact that $N$ could have two $N$-conjugacy class sizes cannot be deduced from the property that $N$ has exactly two $G$-conjugacy class sizes. Some effort has been made in this direction, and in [3] the nilpotency of $N$ is shown under the additional hypothesis that $N$ contains some Sylow $p$-subgroup of $G$ for some prime $p$. In this paper we extend the result with complete generality.

Theorem A. If $N$ is a normal subgroup of a group $G$ and the size of any $G$ conjugacy class contained in $N$ is 1 or $m$, for some integer $m$, then $N$ is nilpotent. More precisely, $N$ is abelian or $N$ is the direct product of a nonabelian p-group $P$ by a central subgroup of $G$. In this case, $P /(\mathbf{Z}(G) \cap P)$ has exponent $p$.

The proof of the nilpotency is clearly divided into two parts. The first part is simpler and deals with the case in which $N$ is solvable (in fact, it is enough to suppose that $\mathbf{F}(N)>\mathbf{Z}(N)$ ). The second part, when $N$ is not solvable, relies on the classification of the finite simple groups by means of a result on $C P$-groups, that is, on groups having all elements of prime power order.

Received by the editors June 3, 2010 and, in revised form, July 14, 2010.

2010 Mathematics Subject Classification. Primary 20E45, $20 \mathrm{D} 15$.

This work is part of the first author's Ph.D. thesis and is partially supported by Proyecto MTM2007-68010-C03-03 and by Proyecto GV-2009-021.

The second author is also supported by grant Fundació Caixa-Castelló P11B2008-09.

(C)2010 American Mathematical Society

Reverts to public domain 28 years from publication 


\section{Proofs}

If $x$ is any element of a group $G$, we denote by $x^{G}$ the conjugacy class of $x$ in $G$ and $\left|x^{G}\right|$ is called the index or the class size of $x$ in $G$. The rest of the notation is standard. We begin by showing the following result whose techniques are elementary.

Theorem 1. Let $N$ be a normal subgroup of a group $G$ such that the size of any $G$-conjugacy class contained in $N$ is 1 or $m$, for some integer $m$. Then either $N$ is abelian or $N /(N \cap \mathbf{Z}(G))$ is a CP-group.

Proof. We will denote by $\bar{G}=G /(\mathbf{Z}(G) \cap N)$ and we will use bars to work in this factor group. Suppose that there exists $g \in N$ such that the order of $\bar{g}$ is not a prime power and we will work to get a contradiction. Then, there exist at least two distinct primes $p$ and $q$ such that $g_{p}$ and $g_{q}$, that is, the $p$-part and $q$-part of $g$ respectively, are non-central elements. By hypothesis, $C_{G}(g)=C_{G}\left(g_{p}\right)=C_{G}\left(g_{q}\right)$. We continue by the following steps.

Step 1. $C_{N}(g) \leq \mathbf{Z}\left(C_{G}(g)\right)$.

Let $w$ be a $q^{\prime}$-element of $C_{N}(g)=C_{N}\left(g_{q}\right)$ and suppose that it is noncentral in $G$. Then

$$
C_{G}\left(g_{q} w\right)=C_{G}\left(g_{q}\right) \cap C_{G}(w)=C_{G}\left(g_{q}\right)=C_{G}(w)
$$

by the hypothesis of the theorem. Hence, $w \in \mathbf{Z}\left(C_{G}\left(g_{q}\right)\right)=\mathbf{Z}\left(C_{G}(g)\right)$. Similarly, one can obtain that if $t$ is a $p^{\prime}$-element of $C_{N}(g)=C_{N}\left(g_{p}\right)$, then $t \in \mathbf{Z}\left(C_{G}(g)\right)$. As a consequence, we conclude that any element $z \in C_{N}(g)$ belongs to $\mathbf{Z}\left(C_{G}(g)\right)$.

Step 2. If $z \in N-\mathbf{Z}(G)$ is such that $C_{G}(g) \neq C_{G}(z)$, then $C_{N}(g) \cap C_{N}(z)=$ $Z(G) \cap N$. Furthermore, either $N$ is abelian or $\mathbf{Z}(N)=\mathbf{Z}(G) \cap N$.

Let $z \in N-\mathbf{Z}(G)$ be such that $C_{G}(g) \neq C_{G}(z)$. Suppose that there exists $a \in C_{N}(g) \cap C_{N}(z)-\mathbf{Z}(G)$. By Step 1, we have $a \in \mathbf{Z}\left(C_{G}(g)\right)$. Therefore, $C_{G}(g)=C_{G}(a)$ by our assumptions. Thus, $z \in C_{N}(a)=C_{N}(g) \leq \mathbf{Z}\left(C_{G}(g)\right)$, using Step 1 again. Consequently, $C_{G}(g) \leq C_{G}(z)$ and so $C_{G}(g)=C_{G}(z)$, which is a contradiction. Hence, $C_{N}(g) \cap C_{N}(z)=\mathbf{Z}(G) \cap N$. Suppose now that $C_{G}(z)=C_{G}(g)$ for every $z \in N-\mathbf{Z}(G)$. Then $N=C_{N}(z)$ for all $z \in N$; that is, $N$ is abelian. Otherwise, there exists some $z \in N-\mathbf{Z}(G)$ such that $C_{G}(g) \neq C_{G}(z)$, and then by the first assertion $\mathbf{Z}(N) \leq C_{N}(g) \cap C_{N}(z)=\mathbf{Z}(G) \cap N$, and we deduce that $\mathbf{Z}(N)=\mathbf{Z}(G) \cap N$.

In the rest of the proof, we may assume that $N$ is not abelian (otherwise the theorem is proved), and thus we assume that $\mathbf{Z}(N)=\mathbf{Z}(G) \cap N$.

Step 3. We have $C_{\bar{G}}(\bar{g})=\overline{C_{G}(g)}$. In particular, $C_{\bar{N}}(\bar{g})=\overline{C_{N}(g)}$.

We clearly have $\overline{C_{G}(g)} \leq C_{\bar{G}}(\bar{g}) \leq C_{\bar{G}}\left(\overline{g_{q}}\right)$ and hence $\left|C_{\bar{G}}(\bar{g})\right|$ divides $\left|C_{\bar{G}}\left(\bar{g}_{q}\right)\right|$. On the other hand, if $\bar{y}$ is an $r$-element of $C_{\bar{G}}\left(\bar{g}_{q}\right)$, with $r \neq q$, then $\left[y, g_{q}\right] \subseteq Z(G)$. If $o(y)=k$, then $1=\left[y^{k}, g_{q}\right]=\left[y, g_{q}^{k}\right]$. Hence, $y \in C_{G}\left(g_{q}^{k}\right)=C_{G}\left(g_{q}\right)=C_{G}(g)$ and $\bar{y} \in \overline{C_{G}(g)}$. Consequently,

$$
\left|C_{\bar{G}}\left(\bar{g}_{q}\right)\right|_{r} \leq\left|\overline{C_{G}(g)}\right|_{r}
$$

As $\left|\overline{C_{G}(g)}\right|_{r}$ divides $\left|C_{\bar{G}}(\bar{g})\right|_{r}$, we conclude that $\left|C_{\bar{G}}(\bar{g})\right|_{r}=\left|\overline{C_{G}(g)}\right|_{r}$ for each prime $r \neq q$. Arguing similarly for the prime $p$, we obtain $\left|C_{\bar{G}}(\bar{g})\right|_{r}=\left|\overline{C_{G}(g)}\right|_{r}$ for every 
prime $r \neq p$. Hence, $\left|C_{\bar{G}}(\bar{g})\right|=\left|\overline{C_{G}(g)}\right|$ and $C_{\bar{G}}(\bar{g})=\overline{C_{G}(g)}$. The second assertion is an immediate consequence.

We remark that the above three steps hold for every conjugate of $g$ in $G$.

Step 4. Conclusion.

First, we claim that there exists some $x \in N$ such that $\bar{g}^{\bar{N} \cap \overline{C_{N}(x)}}=\emptyset$. Suppose that for every $x \in N-\mathbf{Z}(G)$, there exists some $n \in N$ such that $g^{n} \in C_{N}(x)$. Then

$$
N \subseteq \bigcup_{n \in N} C_{N}(g)^{n}
$$

and this implies that $N=C_{N}(g)$ and so $g \in \mathbf{Z}(N)=\mathbf{Z}(G) \cap N$, a contradiction. Thus the claim is proved.

Now, the subgroup $\overline{C_{N}(x)}$ operates on $\bar{g}^{\bar{N}}$ by conjugation. Moreover, no element in $\overline{C_{N}(x)}$ distinct from 1 centralises any element in $\bar{g}^{\bar{N}}$. In fact, if there is some $1 \neq$ $\bar{h} \in \overline{C_{N}(x)}$ which fixes some $\overline{g^{t}}$ for some $t \in N$, it follows that $\bar{h} \in \overline{C_{N}\left(g^{t}\right)} \cap \overline{C_{N}(x)}$, and, by applying Step 2 to $g^{t}$, we deduce that $C_{G}\left(g^{t}\right)=C_{G}(x)$, a contradiction. Hence, all orbits of $\overline{C_{N}(x)}$ on $\bar{g}^{\bar{N}}$ have the same length, that is, $\left|\overline{C_{N}(x)}\right|$, and this implies that $\left|\overline{C_{N}(x)}\right|$ divides $\left|\bar{g}^{N}\right|=\left|\bar{N}: \overline{C_{N}(g)}\right|$ by applying Step 3. Therefore, $\left|\overline{C_{N}(g)}\right|$ divides $\left|\bar{N}: \overline{C_{N}(x)}\right|=\left|x^{N}\right|$, which implies that $\left|\overline{C_{N}(g)}\right|$ divides $\left|x^{G}\right|=$ $\left|g^{G}\right|=\left|\bar{g}^{\bar{G}}\right|$ by Step 3 again.

On the other hand, we claim that $\overline{C_{N}(g)}$ operates without fixed points on $\bar{g}^{\bar{G}}$ $\bar{g}^{\bar{G}} \cap \overline{C_{N}(g)}$ by conjugation. If some $\bar{w} \in \overline{C_{N}(g)}$ distinct from 1 fixes some $\overline{g^{t}}$ for some $t \in G$, then $\bar{w} \in C_{\bar{G}}\left(\bar{g}^{\bar{t}}\right)=\overline{C_{G}\left(g^{t}\right)}$ by applying Step 3 to $g^{t}$. Therefore, $\bar{w} \in \overline{C_{N}(g)} \cap \overline{C_{N}\left(g^{t}\right)}$, and $C_{G}(g)=C_{G}\left(g^{t}\right)$ by Step 2. Thus, $\bar{g}^{\bar{t}}=\overline{g^{t}} \in \overline{C_{N}(g)} \cap \bar{g}^{\bar{G}}$. As a consequence, $\left|\overline{C_{N}(g)}\right|$ divides $\left|\bar{g}^{\bar{G}}-\bar{g}^{\bar{G}} \cap \overline{C_{N}(g)}\right|=\left|\bar{g}^{\bar{G}}\right|-\left|\bar{g}^{\bar{G}} \cap \overline{C_{N}(g)}\right|$. Finally, we conclude that $\overline{C_{N}(g)}$ also divides $\left|\bar{g}^{\bar{G}} \cap \overline{C_{N}(g)}\right|$, which is not possible because

$$
0<\left|\bar{g}^{\bar{G}} \cap \overline{C_{N}(g)}\right|<\left|\overline{C_{N}(g)}\right| .
$$

This contradiction shows that any element of $\bar{G}$ has prime power order.

Corollary 2. Let $N$ be a normal subgroup of a group $G$ such that the size of any $G$-conjugacy class contained in $N$ is 1 or $m$, for some integer $m$. Then $N / \mathbf{Z}(N)$ is a CP-group.

Proof. This is trivial from Theorem 1.

In order to prove the nilpotency of $N$ in Theorem A, we will make use of the following results.

Lemma 3. Let $G$ be a $\pi$-separable group. The size of the conjugacy class of every $\pi$-element of $G$ is a $\pi$-number if and only if $G=H \times K$, where $H$ and $K$ are a Hall $\pi$-subgroup and a $\pi$-complement of $G$, respectively.

Proof. See Lemma 8 of [1] for instance.

Lemma 4. Let $P \times Q$ be the direct product of a p-group $P$ and a $p^{\prime}$-group $Q$ and suppose that $P \times Q$ acts on a p-group $G$. If $C_{G}(P) \subseteq C_{G}(Q)$, then $Q$ acts trivially on $G$.

Proof. This is Thompson's $P \times Q$-Lemma. See for instance 8.2 .8 of $[9]$. 
Lemma 5. Suppose that $G$ is a solvable group and that any $x \in \mathbf{O}_{q}(G)$ has q-index in $G$ for every prime $q$. Then $G$ is nilpotent.

Proof. We argue by induction on the order of $G$. The hypotheses are certainly inherited by normal subgroups of $G$, so every proper normal subgroup of $G$ is nilpotent. If $G$ is not nilpotent, this implies that $\mathbf{F}(G)$ is a maximal normal subgroup of $G$. This means that $G / \mathbf{F}(G)$ is a cyclic group of order $p$ for some prime $p$, so $|G: \mathbf{F}(G)|=p$. Now, if $q \neq p$ is any prime dividing $|\mathbf{F}(G)|$, we have that any $q$-element of $G$ has $q$-index. By Lemma 3, we have $G=Q \times H$, where $H$ is a $q$-complement of $G$, and by induction, we conclude that $H$, and accordingly $G$, are nilpotent, which is a contradiction.

We are going to make use of the structure of finite $C P$-groups. The structure of finite solvable $C P$-groups was given by $\mathrm{H}$. Higman fifty years ago (4]), and the structure of nonsolvable $C P$-groups and the classification of the simple $C P$-groups have been recently obtained by H. Heineken in [5].

Theorem 6. If $G$ is a finite, nonsolvable CP-group, then there are normal subgroups $B, C$ of $G$ such that $1 \subseteq B \subseteq C \subseteq G$ and $B$ is a 2 -group, $C / B$ is nonabelian and simple, and $G / C$ is a p-group for some prime $p$ and cyclic or generalized quaternion.

Proof. This is the main part of Proposition 2 of [5].

Theorem 7. If $G$ is a finite nonabelian simple $C P$-group, then $G$ is isomorphic to one of the following groups: $L_{2}(q)$, for $q=5,7,8,9,17, L_{3}(4), S z(8)$ or $S z(32)$.

Proof. This is Proposition 3 of [5].

Theorem 8. Suppose that $N$ is a normal subgroup of a group $G$ and that the size of any $G$-conjugacy class contained in $N$ is 1 or $m$, for some integer $m$. Then $N$ is nilpotent.

Proof. We argue by induction on the order of $N$. Let $r$ and $q$ be any two primes dividing $|N|$. Let $x$ be any $r$-element of $N$ such that $x \notin \mathbf{Z}(G)$ and take $Q$ to be a Sylow $q$-subgroup of $C_{G}(x)$. Let us consider the action of $Q \times\langle x\rangle$ on $Q_{0}=\mathbf{O}_{q}(N)$. We claim that $C_{Q_{0}}(Q) \subseteq C_{Q_{0}}(x)$. In fact, if $z \in C_{Q_{0}}(Q)$ is noncentral in $G$, then $\langle Q, z\rangle \leq C_{G}(z)<G$. However, by hypothesis, $\left|C_{G}(z)\right|_{q}=\left|C_{G}(x)\right|_{q}=|Q|$, so in particular $z \in Q \cap Q_{0} \subseteq C_{Q_{0}}(x)$. We can apply Lemma 4 and get $x \in C_{N}\left(\mathbf{O}_{q}(N)\right)$. This shows that for any prime $q$, we have that any element lying in $\mathbf{O}_{q}(N)$ has $q$-index in $N$. Now, if $\mathbf{Z}(N)_{q}<\mathbf{O}_{q}(N)$ for some prime $q$, we take an element $w \in \mathbf{O}_{q}(N)-\mathbf{Z}(N)$ and we have $N=C_{N}(w) Q_{w}$ for some $q$-subgroup $Q_{w}$ of $N$. We show that $C_{N}(w)$ is nilpotent. For any $q^{\prime}$-element $y \in C_{N}(w)$, by applying the hypothesis, we have

$$
C_{G}(y w)=C_{G}(w) \cap C_{G}(y)=C_{G}(w) \subseteq C_{G}(y) .
$$

In particular $y \in \mathbf{Z}\left(C_{N}(w)\right)$, which means that $C_{N}(w)$ factorizes as the product of a $q$-group by an abelian subgroup, and hence it is nilpotent as wanted. It follows that $N$ is solvable since it is a product of two nilpotent groups, and thus we can apply Lemma 5 to conclude that $N$ is nilpotent, so the theorem is proved.

Therefore, we can assume for the rest of the proof that $\mathbf{F}(N)=\mathbf{Z}(N)$, so $N$ is nonsolvable, and we will show that this leads to a contradiction. We know by Corollary 2 that $\bar{N}=N / \mathbf{Z}(N)$ is a $C P$-group. By Theorem 6 , there exist $\bar{B}$ and $\bar{C}$ 
subgroups of $\bar{N}$ such that $\bar{B}$ is a 2-group, $\bar{C} / \bar{B}$ is a nonabelian simple group, and $\bar{N} / \bar{C}$ is a $p$-group for some prime $p$. As $\mathbf{F}(N / \mathbf{Z}(N))=1$, we certainly have $\bar{B}=1$. On the other hand, note that $\bar{C}=\mathbf{O}^{p}(\bar{N})$, so in particular $\bar{C}$ is characteristic in $\bar{N}$. Furthermore, since $\mathbf{Z}(N)$ is characteristic in $N$, we conclude that $C$ is normal in $G$. Then, by the inductive hypothesis, we can assume that $\bar{N}$ is a nonabelian simple $C P$-group. If $N^{\prime}<N$, then $N^{\prime}$ would be nilpotent by induction, so $N$ would be solvable, a contradiction. Hence $N^{\prime}=N$, and thus $N$ is a quasi-simple group.

Now, we claim that $|\bar{N}|$ divides $m$. As any element of $\bar{N}$ has prime power order, we can take a noncentral $p$-element $x \in N$ for any prime $p$ dividing $|\bar{N}|$ and notice that we can factorize $C_{N}(x)=C_{N}(x)_{p} \times \mathbf{Z}(N)_{p^{\prime}}$, where $C_{N}(x)_{p}$ is a Sylow $p$-subgroup of $C_{N}(x)$. Then

$$
\left|x^{N}\right|_{p^{\prime}}=\left|N: C_{N}(x)\right|_{p^{\prime}}=|\bar{N}|_{p^{\prime}} .
$$

On the other hand, $\left|x^{N}\right|_{p^{\prime}}$ divides $m_{p^{\prime}}$, so by considering all primes we have that $|\bar{N}|$ must divide $m$, as claimed.

Now, the fact that every element of $N$ is central in $G$ or lies in a $G$-conjugacy class of size $m$ implies that

$$
|N|=|\mathbf{Z}(G) \cap N|+m k,
$$

for some integer $k$. By the above paragraph, we deduce that $|\bar{N}|$ divides $\mid \mathbf{Z}(G) \cap$ $N \mid$, so in particular, it divides $|\mathbf{Z}(N)|$. As $N$ is a quasi-simple group, if $S$ is the associated simple group to $N$, then it can be assumed that $\mathbf{Z}(N) \subseteq M(S)$, where $M(S)$ is the Schur multiplier of $S$. One can easily check (for instance in [2]) that $M(S)$ has order 1, 2, 6 or 48 for the simple groups $S$ appearing in the list of Theorem 7. In all cases, the fact that $|\bar{N}|=|S|$ divides $|M(S)|$ provides the final contradiction.

Corollary 9. Suppose that $N$ is a normal subgroup of a group $G$ such that the size of any $G$-conjugacy class contained in $N$ is 1 or $m$, for some integer $m$. Then $N$ is abelian or $N=P \times A$, with $P$ a p-group and $A$ central in $G$.

Proof. We know that $N$ is nilpotent by Theorem 8 . If $N$ is not abelian, then by applying Theorem 1, we have that $N /(\mathbf{Z}(G) \cap N)$ is a $p$-group for some prime $p$, and then the result follows.

Examples for the two cases appearing in the above corollary can be easily constructed. Let $N$ be an abelian group of odd order and let $\alpha$ be the involutory automorphism of $N$. Then $N$ is an abelian normal subgroup of $G=N\langle\alpha\rangle$ such that the $G$-classes contained in $N$ have size 1 or 2 . On the other hand, let $Q$ be the quaternion group of order 8 and $\beta \in \operatorname{Aut}(Q)$ of order 3. If $G=Q\langle\beta\rangle$, then the $G$-classes contained in $Q$ have size 1 or 6 . This is an example of a nonabelian normal $p$-subgroup of $G$ with exactly two $G$-class sizes.

Several authors, first Isaacs ([7]) and later A. Mann ([10]) or L. Verardi ([1]), have independently proved that if $G$ is a $p$-group with exactly two class sizes, then the exponent of $G / \mathbf{Z}(G)$ is $p$. We are going to extend this result for a normal $p$-subgroup $P$ with two $G$-class sizes, and in particular we provide an alternative proof for the case $P=G$. The approach consists in defining an appropriate normal abelian subgroup contained in $P$ which satisfies certain properties. This construction is inspired by the proof of Proposition 2.2 in [ 8 . We will also need the following recent result due to Isaacs. 
Lemma 10. Let $K \unlhd G$, where $G$ is an arbitrary finite group and $K$ is abelian. Let $x$ be a noncentral element of $G$, and let $y=[t, x]$ for some element $t \in K$. Then $\left|C_{G}(y)\right|>\left|C_{G}(x)\right|$, and so the $G$-class of $y$ is smaller than that of $x$.

Proof. This is exactly Lemma 1 of $[6]$.

Theorem 11. Suppose that $P$ is a nonabelian normal p-subgroup of a group $G$ such that $P$ has only two $G$-conjugacy class sizes. Then $P /(\mathbf{Z}(G) \cap P)$, and in particular $P / \mathbf{Z}(P)$, has exponent $p$.

Proof. We assume that the theorem is untrue and fix some element $x \in P$ such that $x^{p} \notin Z(G)$. Write $Z_{1}=\mathbf{Z}(P)$. Since $P$ is nonabelian, we define $Z_{2} / Z_{1}=\mathbf{Z}\left(P / Z_{1}\right)$. Notice that $Z_{1}<Z_{2}$ and that $Z_{2} \unlhd G$. Let $T_{2}=C_{P}\left(Z_{2}\right)<C_{P}\left(Z_{1}\right)=P$ and observe also that $T_{2} \unlhd G$. The proof has been divided into several steps.

Step 1. If $z \in P-T_{2}$, then $z^{p} \in \mathbf{Z}(G)$. Consequently, $x \in T_{2}$.

By hypothesis $z \notin C_{P}\left(Z_{2}\right)$, so there exists some $y \in Z_{2}$ such that $1 \neq[y, z] \in Z_{1}$. Now, if we consider $z^{p}$, by the hypotheses of the theorem we have two possibilities: either $z^{p} \in \mathbf{Z}(G)$ or $C_{G}\left(z^{p}\right)=C_{G}(z)$. We show that the second case is not possible. Let $p^{a}$ be the order of $[y, z]$. As $[y, z]$ is central in $P$, we have

$$
1=[y, z]^{p^{a}}=\left[y^{p^{a-1}}, z^{p}\right]
$$

so $y^{p^{a-1}} \in C_{G}\left(z^{p}\right)=C_{G}(z)$, which yields to $1=\left[y^{p^{a-1}}, z\right]=[y, z]^{p^{a-1}}$, and this is the required contradiction. Then $z^{p} \in \mathbf{Z}(G)$ and in particular, $x$ must lie in $T_{2}$.

Step 2. There exists an abelian subgroup $T \unlhd G$ with $Z_{1} \subseteq T \subseteq P$ such that if $z \in P-T$, then $z^{p} \in \mathbf{Z}(G)$. As a consequence, $x \in T$.

If $T_{2}$ is abelian, we can take $T=T_{2}$ and the step is proved. So we assume that $T_{2}$ is not abelian, and we may define $Z_{3}$ by means of $Z_{3} / \mathbf{Z}\left(T_{2}\right)=\mathbf{Z}\left(T_{2} / \mathbf{Z}\left(T_{2}\right)\right) \neq 1$ and also define $T_{3}=C_{T_{2}}\left(Z_{3}\right)<T_{2}$. Notice that $Z_{1} \subseteq T_{3}$. Furthermore, as $Z_{3} / \mathbf{Z}\left(T_{2}\right)$ is characteristic in $T_{2} / \mathbf{Z}\left(T_{2}\right)$, and $\mathbf{Z}\left(T_{2}\right)$ is characteristic in $T_{2}$, we deduce that $Z_{3}$ is characteristic in $T_{2}$, so $Z_{3}$ and accordingly $T_{3}$ are normal subgroups in $G$.

Now, we show that if $z \in T_{2}-T_{3}$, then $z^{p} \in \mathbf{Z}(G)$. As $z \notin C_{P}\left(Z_{3}\right)$, there exists some $y \in Z_{3}$ such that $1 \neq[y, z] \in \mathbf{Z}\left(T_{2}\right)$. Arguing as in Step 1 , if $C_{G}\left(z^{p}\right)=C_{G}(z)$ we get a contradiction, so $z^{p} \in \mathbf{Z}(G)$. Therefore, for any $z \in P-T_{3}$, we have $z \in P-T_{2}$ or $z \in T_{2}-T_{3}$, and both cases yield to $z^{p} \in \mathbf{Z}(G)$.

Thus, if $T_{3}$ is abelian, we take $T=T_{3}$ and the step is proved. Otherwise, we can argue as we have done with $T_{2}$ and construct from $Z_{3}$ the subgroups $Z_{4}$ and $T_{4}=C_{P}\left(Z_{4}\right)$, which satisfy that $z^{p} \in \mathbf{Z}(G)$ for any $z \in P-T_{4}$. This method provides a properly descendant series of subgroups $T_{i} \unlhd G$, with $Z_{1} \subseteq T_{i} \subseteq P$, and satisfying the property that $z^{p} \in \mathbf{Z}(G)$ for any $z \in P-T_{i}$. As $Z_{1}$ is abelian, we can get an abelian $T_{i}$ for some $i$, and thus $T=T_{i}$ is the desired subgroup.

Step 3. If $z \in T$ and $z^{p} \notin \mathbf{Z}(G)$, then $C_{P}(z)=T$. In particular $C_{P}(x)=T$.

As $T$ is abelian, we have $T \subseteq C_{P}(z)$. Suppose that $y \in C_{P}(z)-T$. By Step 2, we know that $y^{p} \in \mathbf{Z}(G)$, so $(z y)^{p}=z^{p} y^{p} \notin \mathbf{Z}(G)$. Again by Step 2, we have $z y \in T$, so $y \in T$, a contradiction.

Step 4. If $\bar{G}$ denotes $G /(\mathbf{Z}(G) \cap P)$, then $\overline{C_{P}(x)}=C_{\bar{P}}(\bar{x})$. 
Let $y \in P$ such that $\bar{y} \in C_{\bar{P}}(\bar{x})$. If $y^{p} \notin Z(G)$, then by Step 2, $y \in T$, and by Step 3, we have $C_{P}(x)=T$, so $y \in C_{P}(x)$. If $y^{p} \in \mathbf{Z}(G)$, then $(x y)^{p}=x^{p} y^{p} \notin$ $\mathbf{Z}(G)$. By Step 2, we have $x y \in T$, which implies again $y \in T$. This proves that $C_{\bar{P}}(\bar{x}) \subseteq \overline{C_{P}(x)}$, and the other containment is obvious.

Step 5. Conclusion.

Let $g \in P-T$ and consider $y=[x, g]$. Since $T$ is abelian, we can apply Lemma 10 and get that the $G$-class size of $y \in P$ is smaller than that of $g$. This forces $y$ to be central in $G$, and as a consequence $\bar{g} \in C_{\bar{P}}(\bar{x})=\overline{C_{P}(x)}$ by Step 4 . Therefore, $g \in C_{P}(x)=T$, which is a contradiction. This shows that the element $x$ cannot exist, so $\bar{P}$ has exponent $p$, and the proof finishes.

\section{REFERENCES}

[1] A. Beltrán and M.J. Felipe, Prime powers as conjugacy class lengths of $\pi$-elements. Bull. Austral. Math. Soc. 69 (2004), 317-325. MR2051367 (2005a:20045)

[2] J.H. Conway, R.T. Curtis, S.P. Norton, R.A. Parker, and R.A. Wilson, Atlas of Finite Groups, Clarendon Press, Oxford University Press, 1985. MR 827219 (88g:20025)

[3] X. Guo, X. Zhao, On the normal subgroups with exactly two $G$-conjugacy class sizes. Chin. Ann. Math. B 30 (2009), 427-432. MR2529448 (2010g:20033)

[4] G. Higman, Finite groups in which every element has prime power order, Journal of the London Mathematical Society 32 (1957), 335-342. MR0089205 (19:633d)

[5] H. Heineken, On groups all of whose elements have prime power order. Math. Prod. R. Ir. Acad. 106A (2006), no. 2, 191-198. MR2266826 (2008a:20062)

[6] I.M. Isaacs, Subgroups generated by small classes in finite groups. Proc. Amer. Math. Soc. 136 (2008), 2299-2301. MR2390495 (2009b:20037)

[7] I.M. Isaacs, Groups with many equal classes. Duke Math. J. 37 (1970), 501-506. MR 0263909 $(41: 8508)$

[8] N. Itô, On finite groups with given conjugate types. I. Nagoya Math. J. 6 (1953), 17-28. MR0061597 (15:851c)

[9] H. Kurzweil and B. Stellmacher, The Theory of Finite Groups. An Introduction. SpringerVerlag, New York, 2004. MR2014408 (2004h:20001)

[10] A. Mann, Conjugacy classes in finite groups. Israel J. Math. 31 (1978), 78-84. MR0506901 $(58: 22296)$

[11] L. Verardi, On groups whose noncentral elements have the same finite number of conjugates. Boll. Un. Mat. Ital. A (7) 2 (1988), 391-400. MR.966923 (89k:20037)

Departamento de Matemática Aplicada, Universidad Politécnica de Valencia, 46022 VALENCIA, SPAin

E-mail address: ealemany@mat.upv.es

Departamento de Matemáticas, Universidad Jaume I, 12071 Castellón, Spain

E-mail address: abeltran@mat.uji.es

Instituto Universitario de Matemática Pura y Aplicada, Universidad Politécnica de VAlencia, 46022 VAlencia, Spain

E-mail address: mfelipe@mat.upv.es 